\title{
Differences in allele frequencies of autosomal dominant hypercholesterolemia SNPs in the Malaysian population
}

\author{
Livy Alex, Jagdish Kaur Chahil, Say Hean Lye, Pramod Bagali and Lian Wee Ler
}

Hypercholesterolemia is caused by different interactions of lifestyle and genetic determinants. At the genetic level, it can be attributed to the interactions of multiple polymorphisms, or as in the example of familial hypercholesterolemia (FH), it can be the result of a single mutation. A large number of genetic markers, mostly single nucleotide polymorphisms (SNP) or mutations in three genes, implicated in autosomal dominant hypercholesterolemia (ADH), viz APOB (apolipoprotein B), LDLR (low density lipoprotein receptor) and PCSK9 (proprotein convertase subtilisin/kexin type-9), have been identified and characterized. However, such studies have been insufficiently undertaken specifically in Malaysia and Southeast Asia in general. The main objective of this study was to identify ADH variants, specifically ADH-causing mutations and hypercholesterolemia-associated polymorphisms in multiethnic Malaysian population. We aimed to evaluate published SNPs in ADH causing genes, in this population and to report any unusual trends. We examined a large number of selected SNPs from previous studies of $A P O B$, LDLR, PCSK9 and other genes, in clinically diagnosed ADH patients $(n=141)$ and healthy control subjects $(n=111)$.

Selection of SNPs was initiated by searching within genes reported to be associated with ADH from known databases. The important finding was 137 mono-allelic markers $(44.1 \%)$ and 173 polymorphic markers (55.8\%) in both subject groups. By comparing to publicly available data, out of the 137 mono-allelic markers, 23 markers showed significant differences in allele frequency among Malaysians, European Whites, Han Chinese, Yoruba and Gujarati Indians. Our data can serve as reference for others in related fields of study during the planning of their experiments.

Journal of Human Genetics (2012) 57, 358-362; doi:10.1038/jhg.2012.34; published online 26 April 2012

Keywords: $\mathrm{ADH}$; $A P O B ; L D L R$; MAF; Malaysian; monoallelic; PCSK9

\section{INTRODUCTION}

Hypercholesterolemia, the presence of high level of cholesterol in the blood, is the key factor in the development and progression of atherosclerosis, and consequently is the second major cause of coronary artery disease. ${ }^{1}$ Hypercholesterolemia is influenced by many factors including diet, heredity and other lifestyle factors.

The hereditary type of hypercholesterolemia, called FH (OMIM 143890) is clinically characterized by highly elevated level of plasma LDL cholesterol; deposition of cholesterol in peripheral tissues including xanthelasma, arcus senilis and tendon xanthomas; premature or accelerated atherosclerosis; and increased risk of premature coronary heart disease. Genetically, $\mathrm{FH}$ is an autosomal dominant disorder caused mostly by genetic defects found in the three genes involved in cholesterol homeostasis: LDLR (OMIM 606945), APOB (OMIM 107730) and PCSK9 (OMIM 607786). ${ }^{2}$ Cholesterol can be obtained from both diet and biosynthesis in cells. Cholesterol is hydrophobic, and thus depends on lipoproteins for its transport within the bloodstream. Cholesterol, in cholesteryl ester form, is transported in the plasma mostly via low density lipoprotein (LDL). The homeostasis of cholesterol can be regulated by PCSK9. ${ }^{3}$ PCSK9 is a serine protease of proprotein convertase family. ${ }^{4}$ Mature PCSK9 is secreted from the cell into circulation. ${ }^{5-8}$ The circulating PCSK9 can bind to $L D L R$, leading to the co-internalization, followed by the degradation of $L D L R .{ }^{9}$ Studies demonstrated that the overexpression of PCSK9 results in depletion of $L D L R$, and thus a dramatic increase in plasma level of cholesterol. ${ }^{10-12}$ Conversely, the depletion or removal of PCSK9 protein can substantially increase the level of LDLR and decrease the plasma cholesterol. ${ }^{13-16}$

It is very important that haplotype signatures of prominent SNPs of these three genes are studied in all populations rather than inferring or assuming frequencies from the HAPMAP data, where only a few populations are tested. $L D L R$ is a highly polymorphic gene but how relevant is the CHB (Han Chinese in Beijing) frequencies to other similar races in other locations? There may be many more causal variants waiting to be discovered in other populations; for example, most of the $\mathrm{CHB}$ samples from HAPMAP are northern 
Hans. The southern Hans are separated from northern Hans by the Yangtze river. There has been no study so far done that has analyzed the utility of the CHB data in other Asian populations.

A large number of hypercholesterolemia-associated and $\mathrm{ADH}-$ causing genetic variants, mainly SNPs or mutations in the $L D L R$, $A P O B$ and PCSK9 genes have been documented worldwide. ${ }^{17-19}$ These data are invaluable for the purpose of universal screening and management of ADH. Unfortunately, only a handful of such studies have been conducted in Malaysian and related populations, and thus the repertoire of known $\mathrm{ADH}$-associated or unassociated genetic variants are limited. ${ }^{20-23}$ In the present study, in order to identify $\mathrm{ADH}$ variants, specifically $\mathrm{ADH}$-causing mutations and hypercholesterolemia-associated polymorphisms in Malaysian population, we genotyped both healthy subjects and clinically diagnosed $\mathrm{ADH}$ patients for 310 previously documented single nucleotide mutations or SNPs located in $L D L R, A P O B$ and PCSK9 genes. We found that 137 out of 310 markers were devoid of polymorphic allele in the Malaysian population.

\section{MATERIALS AND METHODS}

Instruments/software used: Illumina Bead Station (Illumina Inc., San Diego, CA, USA) Genome studio software (Illumina Inc.,), all accessories related to hybridization work from Illumina Inc.

\section{Study subjects}

Dutch Lipid Clinic Network (DLCN) criteria for the diagnosis of heterozygous FH were employed for the selection of study subjects.$^{24}$ The inclusion and exclusion criteria are given in appendix-A. A total of 140 clinically diagnosed ADH and 111 control subjects were recruited between January 2007 and September 2009 from the University Malaya Medical Center (UMMC) at Kuala Lumpur. The protocol was reviewed and approved by the UMMC's ethics committee (Ref: 546.16:Detection of FH (LDLR AND APOB) mutations amongst Malaysian population).

\section{DNA isolation}

Blood was collected in various vacutainers in the hospital from the clinically diagnosed ADH and control subjects after overnight fasting of 12-14 h and the lavender cap EDTA vacutainers came to the lab for DNA extraction and the others went for biochemical profiling. The genomic DNA from all subjects was extracted from the whole blood using QIAamp DNA Mini Kit (Qiagen GmbH, Hilden, Germany) according to the manufacturer's instruction. The DNA was adjusted to a final concentration of $50 \mathrm{ng} \mathrm{\mu l}^{-1}$ before microarray analysis.

\section{Genetic marker selection, genotyping assay and data analysis}

Following literature review, polymorphisms previously implicated in $\mathrm{ADH}$ with the following attributes were selected: (i) SNPs in genes encoding $L D L R$, $A P O B, P C S K 9$ and a few other associated genes; (ii) SNPs that were known to have functional effects on in vitro assays or were non-synonymous or in regulatory regions. In $L D L R, 75 \%$ of these SNPs were located in exons with $15 \%$ resulting in stop codons whereas $25 \%$ were in the noncoding region. Three publically available databases: British Heart Foundation (BHF) (http:// www.ucl.ac.uk/ldlr/Current/search.php?select_db=LDLR\&srch=all\&page=11), dbSNP (http://www.ncbi.nlm.nih.gov/projects/SNP/) and SNPedia (www.snpedia. com) were referred for the design of the custom probes.

For the design of the microarray chip, single nucleotide mutations and polymorphisms in $A P O B, L D L R$ and PCSK9 genes (GenBank accession numbers for APOB, LDLR and PCSK9 are AY324608, AY324609 and AY829011, respectively) were selected from two publically available databases: British Heart Foundation (BHF, http://www.ucl.ac.uk/ldlr/Current/search.php? select_db=LDLR\&srch=all\&page=11) and dbSNP (http://www.ncbi.nlm.nih.gov/ projects/SNP/)

Illumina ranks SNPs based on an in-built algorithm in the ADT where SNPs scoring below 0.4 have a rank of zero suggesting the probe is not designable by Illumina. A score between 0.4 and 0.6 gives a rank 0.5 whereas a score above
0.6 is ranked 1 and both these ranks technically can be successfully designed as probes (Oligo Pool All (OPA)) by Illumina. Probes were submitted to ADT in the sequence list format. In all, 231 probes had score of 0.5 and 1305 had a score of 1.0

Genotyping was performed using Illumina GoldenGateGenotyping (GGGT) Microarray Assay. The assay has an average 30-fold redundancy for each probe. All assays were performed according to the manufacturer's protocol and were in compliance with MIAME guidelines. ${ }^{25}$ The microarray data were deciphered using GenomeStudio software and statistical analysis was performed using STATA10 (Stata Corp., College Station, TX, USA). HWE is a basic principle of population genetics. Genotype frequencies at any locus remain same in the absence of migration, mutations, natural selection and assortative mating. When these assumptions are not met, it may result in a deviation from the expected. Hence, a HWE analysis of controls was carried out to exclude the non-conforming SNPs.

Natural selection and genetic drift can often cause genetic differences between populations. Human allele frequencies for various genetic variants differ by geographical locations. The genetic diversity for each SNP used in this study was measured using F-statistics. A rough estimate of genetic differentiation can be made by looking up the fixation index (FST) values. Wherever available, FST values were extracted for the SNPs studied.

\section{Verification of genotyping}

Verification for the allele calls by microarray was done by blindly regenotyping some SNPs in a number of subjects randomly selected from both case and control groups, using DNA sequencing.

\section{RESULTS AND DISCUSSION}

The characteristics of the study subjects are detailed in Table 1. Genomic DNA of 111 healthy control subjects (DLCN criteria $<3$ points) and 140 patients diagnosed with $\mathrm{ADH}$ ( $>3$ points) were genotyped. The mean age was $39.9 \pm 0.90$ and $47.0 \pm 0.90$ years for the controls and $\mathrm{ADH}$ cases, respectively. The diversity of Malaysia's population was also reflected in the ethnic composition of the study subjects.

In total, 310 markers from the public databases were studied, including 130, 73 and 107 markers from $A P O B, L D L R$ and PCSK9 genes, respectively. Among them, no minor allele was observed in 137 markers (73 APOB, 28 LDLR and 36 PCSK9 markers) in both our control and ADH populations (Table 2 and Supplementary Table 1). The failure to find a minor allele among the ADH patients suggested that these genetic markers cannot be used as susceptibility marker for this disorder among Malaysians.

The allelic frequencies of the 137 identified mono-allelic genetic markers, in Han Chinese and European populations, if available, were

Table 1 Characteristics of the study subjects

\begin{tabular}{lcc}
\hline & Control, N=111 & Study, N=140 \\
\hline $\begin{array}{lcc}\text { Age (years } \pm \text { s.e.) } \\
\text { Ethnicity }\end{array}$ & $39.9 \pm 0.90$ & $47.0 \pm 0.90$ \\
$\quad$ Malay, $n(\%)$ & $71(64.0)$ & $84(60.0)$ \\
Indian, $n(\%)$ & $22(19.8)$ & $35(25.0)$ \\
Chinese, $n(\%)$ & $18(16.2)$ & $21(15.0)$ \\
Gender & & $67(47.9)$ \\
Female, $n(\%)$ & $65(58.6)$ & $73(52.1)$ \\
Male, $n(\%)$ & $46(41.4)$ & 0 \\
Dutch Lipid Network & & $93(66.4)$ \\
not FH (<3), $n(\%)$ & 0 & $23(16.4)$ \\
possible (3-5), $n(\%)$ & 0 & $24(17.1)$ \\
probable (6-8), $n(\%)$ & 0 & \\
definite (>8), $n(\%)$ & &
\end{tabular}


Table 2 Summary of genetic markers tested

\begin{tabular}{lccc}
\hline Gene & Markers examined & Mono-allelic markers & \% of total \\
\hline APOB & 130 & 73 & 56.2 \\
LDLR & 73 & 28 & 38.4 \\
PCSK9 & 107 & 36 & 33.6 \\
Total & 310 & 137 & - \\
\hline
\end{tabular}

then extracted from dbSNP (http://www.ncbi.nlm.nih.gov/projects/ $\mathrm{SNP} /$ ) and compared with our data (Supplementary Table 1). In total, the allelic frequencies of 84 out of the 137 markers for Han Chinese or European, or both, were available (Supplementary Table 1). Based on these data, we have identified that the allelic frequencies of 23 markers among Malaysians differed from that of either Han Chinese or European, or both (Table 3). Where available, we also extracted the minor allele frequencies (MAF) of major populations listed in HAPMAP viz. YRI (Yoruba tribe of Ibadan), ASW (African ancestry in southwest USA), GIH (Gujarati Indians of Houston), LWK (Luhya in Webuye, Kenya), TSI (Tuscans of Italy) and JPT (Japanese of Tokyo area). Most of these markers have a MAF of $<10 \%$, with the exception of rs61318752 (CHB), rs127208519 (YRI, ASW and LWK), rs10422244 (YRI, ASW and LWK), rs12720828 (CEU and TSI) and rs10199768 (CEU, YRI, ASW, GIH, LWK and TSI). Interestingly, rs61318752 has a reported MAF of $11.4 \%$ among Han Chinese, but was devoid of minor allele among Malaysians even though our Malaysian subjects also included 39 individuals of ethnic Chinese origin. One possible reason for this discrepancy is that almost all Chinese Malaysians are of Southern Chinese origin; and the minor allele of rs61318752 could be absent in population which originates from Southern China. Also, it is of interest to see that rs12720851, rs10422244 and rs17111557 have a MAF in the range of $10-18 \%$ for the African ancestry populations, YRI and LWK showing a positive correlation between the two tribes. From the figures listed, it seems that they both share common antecedents. Joubert et al., ${ }^{26}$ in their study on African ancestry populations also show the average $r^{2}$ between two SNPs of YRI and LWK to be around 0.857. Adjacent SNP-SNP linkage disequilibrium was highly correlated across populations of African ancestry except for the Malawian Population. Geographic distance and inbreeding seem to be the two most common parameters for genetic heterogeneity. Thus, the frequencies of risk alleles of SNPs and mutations defining the susceptibility to $\mathrm{ADH}$ differ significantly between population to population and natural selection and genetic drift are the two commonest factors responsible.

FST is an appropriate index of allele-frequency differentiation, which captures the difference in allele frequency between two populations at any given SNP and ranges from 0 (no differentiation) to 1 (fixed difference between populations). FST values plummet if there is greater gene flow and less of inbreeding. With migrant number influx increasing into each population, it is expected that FST will decrease further but we still see such sharp genetic differences between populations. (At equilibrium between drift and gene flow, $\mathrm{FST}=1 /(1+4 \mathrm{Nm})$, where $\mathrm{Nm}$ is the number of migrants moving into each sub-population. ${ }^{27}$ It is most likely that it is due to selection.) SNPs follow peculiar distribution according to the geographic nature of the selection pressure.

Although FST has a theoretical range of $0-1.0$, the observed maximum is usually much $<1.0$. Wright (1978) suggests the following qualitative guidelines for the interpretation of FST (based on allozyme loci):

- the range 0.0-0.05 may be considered as indicating little genetic differentiation

- the range $0.05-0.15$ indicates moderate genetic differentiation

- the range $0.15-0.25$ indicates great genetic differentiation

- values of FST above 0.25 indicate very great genetic differentiation

The basis of such varied differentiation is still a partial mystery. The types of selection acting on SNPs in a particular population need to be understood better.

Common alleles are shared by all populations. Genetic drift or natural selection reduces heterozygosity by the random loss of alleles. Infinitely large populations will not experience drift whereas small populations will experience major drift. Drift is thus one of the major forces of evolution along with natural selection, mutation, non random mating etc.

The two markers rs41288783 and rs10422244 in $A P O B$ and $L D L R$, respectively, show an FST of $\sim 0.3$, which is quite pronounced and the MAF also correlates as well (Table 3). The frequency of a risk allele discovered in one population is not always a strong predictor of the disease frequency in other populations. Several disease-associated alleles or protective alleles appear to have been driven to high frequency by positive selection in certain human populations.

Out of these 23 SNPs reported in ADH cases, rs1801703, rs12720848, rs568413, rs12713844, rs41288783 lead to non-conservative amino-acid replacements of Val to Met, His to Asn, Cys to Tyr, Asp to His, Pro to Leu, respectively, but to our dismay, we found the wildtype allele present in the Malaysian population, both in cases and controls, proving that either these variations thought to contribute to $\mathrm{ADH}$ susceptibility as seen in other populations is not a cause of concern in this ethnicity or more robust functional assays are expected on these SNPs to ascertain an associative role.

Genetic heterogeneity in any complex disease leads us to hunt for novel genes and the risk alleles therein. Similarly genetic heterogeneity for $\mathrm{ADH}$ among different populations provides the possibility of uncovering other uncharacterized genes that may be involved in the pathogenesis of the disease. For example, in a Mexican population, no PCSK9 mutations were found in one large ADH family that showed positive linkage to the 1p34.1-32 locus; this indicates genes other than PCSK9 in the locus may be involved. ${ }^{28}$ In addition, a Portuguese $\mathrm{ADH}$ study found only $48 \%$ of its total received cases with clinical diagnosis of $\mathrm{ADH}$ had genetic defects on $L D L R, A P O B$ or PCSK9, leaving the other $52 \%$ of $\mathrm{ADH}$ cases with possible undiscovered gene mutations. $^{29}$

In a study by Garcia et al., ${ }^{30}$ the researchers used gene chip (robo arrays) to resequence the coding regions of 10 key genes of lipid metabolism in 80 dyslipidemic cases. In all, 14 non-synonymous and 22 synonymous SNPs were identified, which were confirmed by conventional sequencing. Coding sequence variations thought to be associated with dyslipidemia were also seen in controls. This study is certainly a good evidence or instance for all researchers to understand that work on common variants and causal variant need to be published regardless of how significant or insignificant it may be considered.

There is a dire need for systematic sequencing of all genes involved in cholesterol metabolism in different populations to decipher which sequence variation causes moderate or high elevations of lipoproteins. Normally $10-20 \%$ of SNPs lead to a non-conservative change aminoacid replacement. SNPs are commonly used to study genetics for 
Table 3 The list of mono-allelic markers that are polymorphic in various populations

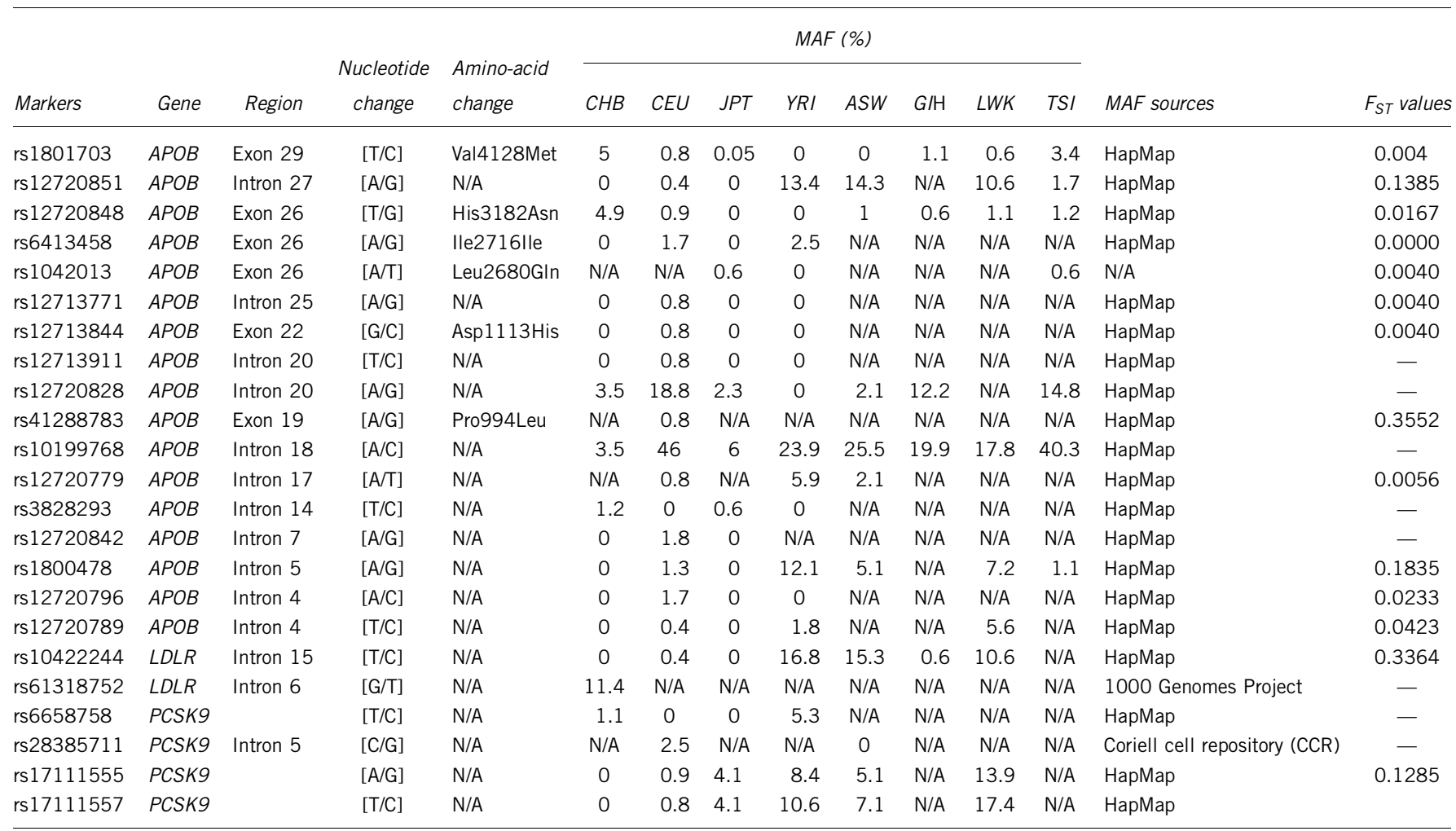

Abbreviation: MAF-minor allele frequency

$\mathrm{F}_{\mathrm{ST}}$ values are from SNP at evolution

CHB-Han Chinese in Beijing, China CEU-Utah residents with Northern and Western European ancestry from the CEPH collection JPT-Japanese in Tokyo, Japan YRI-Yoruba in Ibadan, Nigeria ASWAfrican ancestry in Southwest USA GIH-Gujarati Indians in Houston, Texas LWK-Luhya in Webuye, Kenya TSI-Toscans in Italy

common diseases and predict pharmacological response. The selection of likely informative SNPs in association studies depends on their allele frequencies and on the linkage disequilibrium between SNPs, both of which may show inter ethnic differences.

$\mathrm{Ng}$ et al., ${ }^{31}$ compared the allele frequency distributions of 64 intragenic SNPs of 35 candidate genes for cardiovascular diseases in three populations consisting of 207 Chinese, 858 French and 395 Spanish individuals. In all, 28 of these SNPs from 12 genes were also examined for intragenic linkage disequilibrium. About $20 \%$ of SNPs were restricted to Europeans, being monomorphic in Chinese, among them mostly nonsynonymous coding SNPs and noncoding SNPs. Only $1.6 \%$ of SNPs were specific in Chinese, commensurate with the detection of these SNPs almost exclusively in Caucasians. Similarly, these SNPs were more often rare $(<0.1$ minor allele frequency) in Chinese $(44.3 \%)$ than in Europeans $(31.1 \%)$. The variant allele frequencies and intermarker linkage disequilibriums in terms of $D^{\prime}$ and $\Delta^{2}$ were highly correlated between French and Spanish populations $(r=0.98-0.99, P<0.001)$. However, only moderate correlations of allele frequencies and $D^{\prime}$ were found between the Chinese and the European populations $(r=0.7$ and 0.3 , respectively) despite a high correlation of $\Delta^{2}$ values $(r=0.8) .^{31}$

Hence, it is very suggestive and ethical that we pay utmost care in interpreting meaning of variations in the clinical setting in various ethnicities. The 310 genetic markers studied represent only a small subset of all genetic alterations and diversities in $L D L R, A P O B$ and PCSK9 genes that may potentially affect cholesterol metabolism, homeostasis and $\mathrm{ADH}$ documented thus far. It is known that genetic variants associated with complex diseases vary from population to population. ${ }^{32}$ The different repertoires of genetic markers associated with hypercholesterolemia or causing $\mathrm{ADH}$ are population specific, ${ }^{18}$ more studies are needed to characterize the full range of genetic alterations among Malaysians. By disclosing the identities of these mono-allelic loci, we hope we may contribute to the better understanding of genetic markers associated with cholesterol metabolism and disorders in Malaysian population and provide useful insights.

\section{CONFLICT OF INTEREST}

The authors declare no conflict of interest. The views and interpretations expressed in this paper are those of the authors based on past studies and do not reflect the views of the management sponsoring the project.

\section{ACKNOWLEDGEMENTS}

We greatly appreciate the support of the study subjects and their families who participated in this project. This work was funded by the Ministry of Science, Technology and Innovation (MOSTI), the Government of Malaysia. We thank the staff of Faculty of Medicine (Universiti Malaya), UMMC and INFOVALLEY ${ }^{\circledR}$ for their timely help during the subject recruitment and sample collection. Our special gratitude goes to all the doctors and lab technicians who assisted us during patient recruitment, sample collection and administrative processes. 
1 Toutouzas, K., Drakopoulou, M., Skoumas, I. \& Stefanadis, C. Advancing therapy for hypercholesterolemia. Expert Opin. Pharmacother. 11, 1659-1672 (2010).

2 Soutar, A. K. \& Naoumova, R. P. Mechanisms of disease: genetic causes of familial hypercholesterolemia. Nat. Clin. Pract. Cardiovasc. Med. 4, 214-225 (2007).

3 Mousavi, S. A., Berge, K. E. \& Leren, T. P. The unique role of proprotein convertase subtilisin/kexin 9 in cholesterol homeostasis. J. Intern. Med. 266, 507-519 (2009).

4 Seidah, N. G. \& Prat, A. The proprotein convertases are potential targets in the treatment of dyslipidemia. J. Mol. Med. (Berl.) 85, 685-696 (2007).

5 Cunningham, D., Danley, D. E., Geoghegan, K. F., Griffor, M. C., Hawkins, J. L., Subashi, T. A. et al. Structural and biophysical studies of PCSK9 and its mutants linked to familial hypercholesterolemia. Nat. Struct. Mol. Biol. 14, 413-419 (2007).

6 Naureckiene, S., Ma, L., Sreekumar, K., Purandare, U., Lo, C. F., Huang, Y. et al. Functional characterization of Narc 1, a novel proteinase related to proteinase K. Arch. Biochem. Biophys. 420, 55-67 (2003).

7 Piper, D. E., Jackson, S., Liu, Q., Romanow, W. G., Shetterly, S., Thibault, S. T. et al. The crystal structure of PCSK9: a regulator of plasma LDL-cholesterol. Structure 15, 545-552 (2007).

8 Seidah, N. G., Benjannet, S., Wickham, L., Marcinkiewicz, J., Jasmin, S. B., Stifani, S et al. The secretory proprotein convertase neural apoptosis-regulated convertase 1 (NARC-1): liver regeneration and neuronal differentiation. Proc. Natl Acad. Sci. USA 100, 928-933 (2003).

9 Lambert, G., Charlton, F., Rye, K. A. \& Piper, D. E. Molecular basis of PCSK9 function. Atherosclerosis 203, 1-7 (2009).

10 Lalanne, F., Lambert, G., Amar, M. J. et al. Wild-type PCSK9 inhibits LDL clearance but does not affect apoB-containing lipoprotein production in mouse and cultured cells. J. Lipid Res. 46, 1312-1319 (2005).

11 Maxwell, K. N. \& Breslow, J. L. Adenoviral-mediated expression of PCSK9 in mice results in a low-density lipoprotein receptor knockout phenotype. Proc. Natl Acad. Sci. USA 101, 7100-7105 (2004).

12 Park, S. W., Moon, Y. A. \& Horton, J. D. Post-transcriptional regulation of low density lipoprotein receptor protein by proprotein convertase subtilisin/kexin type $9 a$ in mouse liver. J. Biol. Chem. 279, 50630-50638 (2004).

13 Frank-Kamenetsky, M., GreADHorst, A., Anderson, N. N., Racie, T. S., Bramlage, B., Akinc, A. et al. Therapeutic RNAi targeting PCSK9 acutely lowers plasma cholesterol in rodents and LDL cholesterol in nonhuman primates. Proc. Natl Acad. Sci. USA 105, 11915-11920 (2008).

14 Graham, M. J., Lemonidis, K. M., Whipple, C. P., Subramaniam, A., Monia, B. P. Crooke, S.T. et al. Antisense inhibition of proprotein convertase subtilisin/kexin type 9 reduces serum LDL in hyperlipidemic mice. J. Lipid Res. 48, 763-767 (2007).

15 Rashid, S., Curtis, D. E., Garuti, R., Anderson, N. N., Bashmakov, Y., Ho, Y. K. et al. Decreased plasma cholesterol and hypersensitivity to statins in mice lacking PCSK9. Proc. Natl Acad. Sci. USA 102, 5374-5379 (2005).

16 Zaid, A., Roubtsova, A., Essalmani, R., Marcinkiewicz, J., Chamberland, A., Hamelin, J. et al. Proprotein convertase subtilisin/kexin type 9 (PCSK9): hepatocytespecific low-density lipoprotein receptor degradation and critical role in mouse liver regeneration. Hepatology 48, 646-654 (2008).
17 Abifadel, M., Rabes, J. P., Jambart, S., Halaby, G., Gannagé-Yared, M. H., Sarkis, A et al. The molecular basis of familial hypercholesterolemia in Lebanon: spectrum of LDLR mutations and role of PCSK9 as a modifier gene. Hum. Mutat. 30, E682-E691 (2009).

18 Dos Santos, J. E. \& Zago, M. A. Familial hypercholesterolemia in Brazil. Atheroscler Suppl. 4, 1-2 (2003).

19 Leigh, S. E., Foster, A. H., Whittall, R. A., Hubbart, C. S. \& Humphries, S. E. Update and analysis of the University College London low density lipoprotein receptor familial hypercholesterolemia database. Ann. Hum. Genet. 72, 485-498 (2008).

20 Azian, M., Hapizah, M. N., Khalid, B. A., Khalid, Y., Rosli, A. \& Jamal, R. Use of the denaturing gradient gel electrophoresis (DGGE) method for mutational screening of patients with familial hypercholesterolaemia (ADH) and Familial defective apolipoprotein B100 (FDB). Malays. J. Pathol. 28, 7-15 (2006).

21 Choong, M. L., Koay, E. S., Khoo, K. L., Khaw, M. C. \& Sethi, S. K. Denaturing gradient-gel electrophoresis screening of familial defective apolipoprotein B-100 in a mixed Asian cohort: two cases of arginine3500->tryptophan mutation associated with a unique haplotype. Clin. Chem. 43, 916-923 (1997).

22 Khoo, K. L., Van Acker, P., Tan, H. \& Deslypere, J. P. Genetic causes of familia hypercholesterolaemia in a Malaysian population. Med. J. Malaysia 55, 409-418 (2000).

23 Saha, N., Tay, J. S. \& Chew, L. S. Influence of apolipoprotein B signal peptide insertion/ deletion polymorphism on serum lipids and apolipoproteins in a Chinese population. Clin. Genet. 41, 152-156 (1992).

24 Yuan, G., Wang, J. \& Hegele, R. A. Heterozygous familial hypercholesterolemia: an underrecognized cause of early cardiovascular disease. CMAJ 174, 1124-1129 (2006).

25 Brazma, A., Hingamp, P., Quackenbush, J., Sherlock, G., Spellman, P., Stoeckert, C. et al. Minimum information about a microarray experiment (MIAME)-toward standards for microarray data. Nat. Genet. 29, 365-371 (2001).

26 Joubert, B. R., North, K. E., Wang, Y., Mwapasa, V., Franceschini, N., Meshnick, S.R. et al. Comparison of genome-wide variation between Malawians and African ancestry HapMap populations. J. Hum. Genet. 55, 366-374 (2010).

27 Boyce, A. J. \& Mascie-Taylor, C. G. N. (ed.) Molecular Biology and Human Diversity (Cambridge University Press, Cambridge, UK, 1996).

28 Robles-Osorio, L., Huerta-Zepeda, A., Ordonez, M. L., Canizales-Quinteros, S. Díaz-Villaseñor, A., Gutiérrez-Aguilar, R. et al. Genetic heterogeneity of autosomal dominant hypercholesterolemia in Mexico. Arch. Med. Res. 37, 102-108 (2006)

29 Medeiros, A. M., Alves, A. C. \& Francisco, V. Bourbon M. Update of the Portuguese Familial Hypercholesterolaemia Study. Atherosclerosis 212, 553-558 (2010).

30 Garcia, C. K., Mues, G., Liao, Y., Hyatt, T., Patil, N., Cohen, J.C. et al. Sequence diversity in genes of lipid metabolism. Genome Res. 11, 1043-1052 (2001).

$31 \mathrm{Ng}$, M. C., Wang, Y., So, W. Y., Cheng, S., Visvikis, S., Zee, R. Y. et al. Ethnic differences in the linkage disequilibrium and distribution of single-nucleotide polymorphisms in 35 candidate genes for cardiovascular diseases. Genomics 83, 559-565 (2004).

32 Adeyemo, A. \& Rotimi, C. Genetic variants associated with complex human diseases show wide variation across multiple populations. Public Health Genomics 13, 72-79 (2010).

Supplementary material accompanies the paper on Journal of Human Genetics website (http://www.nature.com/jhg) 